\title{
Psychosoziale Herausforderungen und Bedürfnisse junger Frauen nach onkologischer Behandlung eines Mammakarzinoms
}

Die hohe Inzidenz des Mammakarzinoms bei jung erkrankten Frauen stellt für Betroffene, deren soziales Umfeld und nicht zuletzt deren Behandler sowohl medizinische als auch psychosoziale Herausforderungen dar. Viele Themen werden erst in der Phase des "Survivorships“ nach Abschluss der Therapien sowie in der Nachsorge relevant. Gynäkologen werden zunehmend weniger aufgrund medizinischer Aspekten, dafür mehr wegen psychosozialer Themen (psychische Belastung, Sexualität, Partnerschaft und Familie) konsultiert. Im Folgenden soll näher auf psychosoziale Herausforderungen und Bedürfnisse junger Frauen sowie auf Unterstützungsmöglichkeiten seitens gynäkologischer Behandler eingegangen werden.

\section{Hintergrund}

In Deutschland ist Brustkrebs die häufigste Krebserkrankung der Frau, und jährlich erkranken etwa 70000 Frauen (Zahlen von 2012) neu daran. Es wird geschätzt, dass 2016 etwa 65500 Frauen neu erkrankt sind, was konkret bedeutet, dass jede 8. Frau im Laufe ihres Lebens an Brustkrebs erkrankt. Anders als bei anderen Krebserkrankungen ist das Erkrankungsalter bei Mammakarzinom deutlich niedriger. Fast 30\% (ca. 21000 Frauen) der betroffenen Frauen sind bei Diagnosestellung jünger als 55 Jahre [1].

Die Diagnose Brustkrebs bedeutet die Konfrontation mit einer lebensbedrohlichen $\mathrm{Er}$ krankung, der eigenen Endlichkeit sowie dass die eigene Gesundheit plötzlich nicht mehr selbstverständlich ist. Die Erkrankung und die therapiebedingten körperlichen Strapazen führen häufig zunächst zu einer massiven Verunsicherung sowie emotionaler Überforderung. Aufgrund dessen erfahren viele Patientinnen während der Therapie und nach Abschluss der Behandlungen soziale, emotionale und körperliche Beeinträchtigungen in ihrem Alltag [2,3]. Aktuelle Studien zeigen, dass Frauen mit
Mammakarzinom am häufigsten unter psychischen Belastungen leiden und im Krankheitsverlauf psychische Erkrankungen wie Anpassungsstörung, Depression oder eine Angststörung entwickeln können [4,5]. Insbesondere jüngere Frauen (<50 Jahre) sind durch die Erkrankung und nach Abschluss der Therapien deutlich belasteter als ältere betroffene Frauen (Risikofaktoren einer maladaptiven Krankheitsverarbeitung sind in $>$ Tab. 1 dargestellt) [6].

RISIKOFAKTOREN, DIE BEI JUNGEN PATIENTINNEN ZU EINER ERSCHWERTEN KRANKHEITSBEWÄLTIGUNG FÜHREN KÖNNEN (MODIFIZIERT NACH [20])

Risikofaktoren einer maladaptiven Krankheitsbewältigung

medizinische

- fortgeschrittener Erkrankungsstatus

- aggressive und invasive Therapiemethoden

- tumorbedingte Fatigue

- schwerwiegende Komplikationen oder Wechselwirkungen

- Komorbidität mit anderen Erkrankungen

- positive Familienanamnese

persönliche

- junges Alter

- psychosoziale Bedürfnisse (z. B. Kinderwunsch, Sexualität, finanzielle Probleme)

- fehlende Copingstrategien

- psychische Erkrankung in der Vorgeschichte

- geringes Einkommen/Bildung

soziale

- fehlende soziale Unterstützung

- geringerer Zugang zu Unterstützungsangeboten

- Tabuisierung der Erkrankung

- Arbeitslosigkeit
Die erlebten psychosozialen Belastungen hängen u. a. eng mit den Entwicklungsaufgaben zusammen, die jüngere Frauen trotz der Erkrankung in ihrem Leben durchlaufen und bewältigen müssen. Frauen im Alter von 20-50 Jahren setzen sich mit zentralen Entwicklungsaufgaben auseinander wie z.B. Gestaltung einer tragfähigen Partnerschaft; Etablierung der beruflichen Zukunft; Verantwortung sowie Erziehung der eigenen Kinder oder Übernahme sozialer Verantwortung [7]. All diese Entwicklungsaufgaben werden durch die frühere Erkrankung infrage gestellt und können gemeinsam mit der Vulnerabilität der eigenen Integrität zu erheblichen Belastungen, Ängsten und Sorgen führen $[3,8]$. Diese können die Patientinnen und das soziale Umfeld massiv beeinträchtigen und eine große Herausforderung in der Versorgung und Betreuung junger Patientinnen darstellen. Neben der ärztlichen und gynäkologischen achtsamen Betreuung, ist es von großer Bedeutung, die betroffenen jungen Frauen auf ihrem Weg zu begleiten und ihnen eine Orientierung in ihrem Anpassungsprozess an die Veränderungen der verschiedenen Lebensbereiche aufgrund der Erkrankung und Therapie zu geben. Dabei ist es wichtig wahrzunehmen, wie betroffene Frauen ihre veränderte Lebenssituation erfahren, um dadurch die tatsächlichen Probleme und Bedürfnisse frühzeitig erkennen zu können. Erst dann wird man ihnen und ihren Familien eine professionelle Unterstützung anbieten können. Im Folgenden werden einige psychosoziale Problem- und Belastungsbereiche betroffener junger Frauen nach Abschluss der Therapien und in der Nachsorge herausgearbeitet sowie praktische Fragen zur Exploration inklusive hilfreicher Informationen für Patientinnen dargestellt. 


\section{individuelle Skillsbox}

Emotionsregulation
- Gefühle erkennen
- Akzeptanz
- Veränderung der Reizexposition
- Veränderung der Bewertung
- Aufmerksamkeitslenkung

\section{soziales Umfeld}

- Familie

- Partnerschaft

- freundschaftliche Beziehungen

- Wünsche äußern

- Selbsthilfegruppe

Verein, Kirchengemeinde etc.

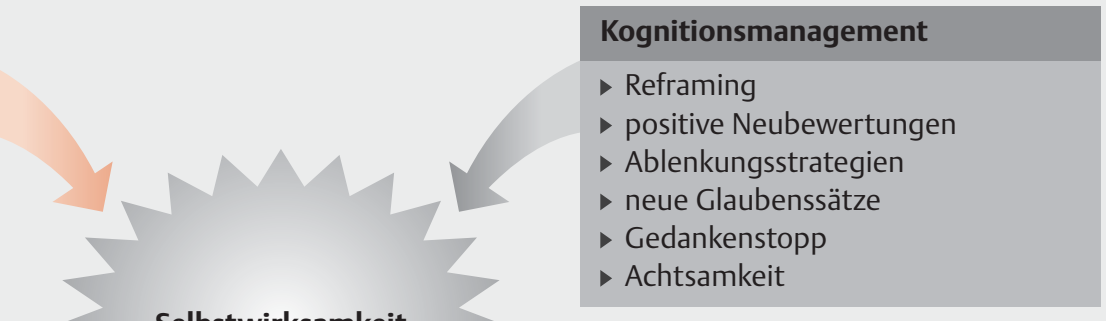

angenehme Aktivitäten

- Spazieren gehen

- Sport, Hobbys

- Theater- oder Kinobesuche

- unterhaltsame Literatur

- Kreuzworträtsel, Sudoku

Lieblingsmusik hören

- Abb. 1 Die individuelle Skillsbox, die in seelischen Notlagen helfen kann.

\section{Psychosoziale Belastungen und Bedürfnisse}

Infolge körperlicher Beeinträchtigungen und Nebenwirkungen durch die Krankheit entstehen häufig psychosoziale Belastungen und Bedürfnisse bei jungen Patientinnen $[6,9]$. Aktuelle Studien zeigen, dass Patientinnen nach Abschluss der Therapien oft unter einer geringeren Lebensqualität und statistisch häufiger unter einer Depression oder Angststörung im Vergleich zur Allgemeinbevölkerung leiden. Patientinnen wünschen sich nach Abschluss der Akutbehandlung häufig eine Unterstützung im Umgang mit psychosozialen Belastungen wie Sexualität, Partnerschaft, Kinderwunsch und Arbeit [6, 9]. Aufgrund langwieriger und aggressiver Therapiemaßnahmen müssen nicht selten - z. T. vorübergehend, z. T. auch auf Dauer - Alltagsaktivitäten aufgegeben werden. Der bisherige Lebensentwurf muss ggf. infrage gestellt sowie Lebensziele überdacht und neu ausgerichtet werden. Diese Veränderung bedeutet häufig eine enorme Herausforderung im Anpassungsprozess an die neue Lebenssituation für junge $\mathrm{Pa}$ tientinnen [2,3]. Wichtig ist es hierbei, dass Ärzte den Patientinnen immer wieder aufzeigen, dass es trotz der Veränderung Dinge gibt, die ihnen gut tun und wichtig für sie sind. Die Bedeutung der eigenen Selbstwirksamkeit anhand einer individuellen „Skillsbox“ mit wohltuenden Aktivitäten, eigenen Fertigkeiten und Fähigkeiten sollten immer wieder herausgearbeitet und aufgezeigt werden ( $\triangleright$ Abb. 1).

Ängste, insbesondere Progredienzängste, sollten normalisiert und validiert werden. Patientinnen erleben es als sehr entlastend, wenn Ängste als eine Art Schutz gesehen werden („reframing“). Beispielhaft sei eine Intervention zur Änderung der Bewertung von Ängsten dargestellt („Säbelzahntigerprinzip“):
- Hilfreich sind Bilder und Geschichten, wie die unserer Vorfahren aus der Steinzeit. Die Angst vor natürlichen Feinden wie dem Säbelzahntiger, ermahnte die Urmenschen zur Vorsicht und Aufmerksamkeit, aber auch zum aktiven Handeln gegen die Gefahr.

Angst kann so als wohlwollender - zumindest aber als tolerierbarer - Begleiter „normalisiert“ werden. Das „Säbelzahntigerprinzip“ entlastet Patientinnen häufig sehr, da sie verstehen, dass Ängste ihnen helfen, achtsam mit sich und ihrem Körper zu sein, um regelmäßig die Nachsorge oder weitere Therapien durchzuführen.

- Tab. 1 Hilfreiche Fragen zum Thema psychosoziale Belastungen und Bedürfnisse.

\begin{tabular}{|l|l|}
\hline Ziel & mögliche Fragen \\
\hline $\begin{array}{l}\text { Klärung und Erfragen } \\
\text { aktueller Belastungs- } \\
\text { bereiche }\end{array}$ & $\begin{array}{l}\text { 1. Gibt es seit der Erkrankung Dinge, die Sie „seelisch“ sehr } \\
\text { belasten? }\end{array}$ \\
\hline $\begin{array}{l}\text { 2. Treten aktuell vermehrt Gefühle der Traurigkeit, Angst oder } \\
\text { Schlafstörungen auf? }\end{array}$ \\
\hline $\begin{array}{l}\text { Normalisierung und } \\
\text { Ealidierung aktueller }\end{array}$ & $\begin{array}{l}\text { 3. Viele Frauen berichten, dass sie seit der Erkrankung oder } \\
\text { nach Abschluss der Therapien häufig traurig oder verzweifelt } \\
\text { sind. Haben Sie dies auch schon bei sich bemerkt? }\end{array}$ \\
\hline $\begin{array}{l}\text { 4. Es ist normal, dass wir nach einer schweren Erkrankung auch } \\
\text { seelische Belastungen entwickeln. Wie erleben Sie die aktu- } \\
\text { elle Situation? }\end{array}$ \\
\hline $\begin{array}{l}\text { Ansprechen von Skills } \\
\text { und Ressourcen }\end{array}$ & $\begin{array}{l}\text { 6. Gibt es Dinge, die Ihnen schon mal in schwierigen Situa- } \\
\text { tionen geholfen haben? }\end{array}$ \\
\hline
\end{tabular}


Depressive Verstimmungen können auch als Trauer(-prozess) um den Verlust der körperlichen Integrität oder der Alltagsaktivtäten benannt werden. Diese Deutung entlastet und hilft den Frauen bei der Krankheitsverarbeitung. Emotionale Reaktionen wie Trauer, Wut, Ängste, Schlafprobleme, Hilflosigkeit und Verzweiflung dürfen auch nach einer Erkrankung auftreten und sind adäquat für die Belastungssituation [8]. Allerdings sollte darauf geachtet werden, ob Patientinnen diese Emotionen und Gefühlszustände alleine bewältigen können oder ob sie professionelle psychotherapeutische/psychoonkologische Behandlung oder eine psychopharmakologische Unterstützung im weiteren Verlauf benötigen ( $\triangleright$ Tab. 1$)$.

\section{Tabuthema Sexualität}

Studien zeigen, dass aufgrund der Behandlungen und Therapien viele junge Patientinnen unter sexuellen Funktionsstörungen sowie Einschränkungen der sexuellen Appetenz leiden. Diese Einschränkungen stellen u. U. eine enorme Belastung für die Patientinnen, deren Partner und die Partnerschaft insgesamt dar $[10,11]$. Durch die Folgen der Therapie kann der weibliche Zyklus gestört sein und Frauen treten frühzeitig in eine Menopause ein, die sich negativ auf die Libido und sexuelle Funktionen auswirken kann. Die Antihormontherapie mit den damit verbundenen Veränderungen (Scheidentrockenheit und verminderte Lubrikation) bringt Appetenzstörung und Schmerzen während des Geschlechtsverkehrs mit sich. Sex nach Krebs ist bei vielen Patientinnen ein Tabuthema, da sie häufig das Gefühl haben, dass sie Schlimmes durchgemacht haben und es ihnen nun nicht zusteht, aufgrund solcher „banalen“ Beschwerden den Gynäkologen aufzusuchen bzw. nach Unterstützung zu fragen [12]. Zusätzlich fühlen sich viele Frauen aufgrund der Therapiefolgen in ihrem Körper durch ihr verändertes Äußeres wie z.B. durch Ablatio mammae, Implantataufbau oder Gewichtszunahme in und mit ihrem Körper unwohl [13]. Je selbstverständlicher und offener ein Arzt mögliche Probleme thematisiert, umso leichter wird es für die Patientin, konkrete Probleme mit dem Körperbild oder Ängste zu äußern und nach Unterstützung zu fra-
- Tab. 2 Hilfreiche Fragen zum Thema Sexualität.

\begin{tabular}{|l|l|l|}
\hline Ziel & mögliche Fragen \\
\hline $\begin{array}{l}\text { Klärung und Förderung } \\
\text { einer offenen Kommunika- } \\
\text { tion }\end{array}$ & $\begin{array}{l}\text { 1. Sie haben ja gerade generell über empfindliche Schleim- } \\
\text { häute gesprochen, wie ist es im Intimbereich? } \\
\text { 2. Ich sehe, dass Sie im Intimbereich sehr trocken sind, ist dies } \\
\text { aktuell ein Problem für Sie? } \\
\text { 3. Können Sie mit Ihrem Partner über sexuelle Beschwerden/ } \\
\text { Belastungen sprechen? }\end{array}$ \\
\hline \begin{tabular}{l} 
4. Hat Ihr Partner Verständnis für Ihre aktuelle Situation? \\
\hline von Hemmschwellen
\end{tabular} & $\begin{array}{l}\text { 5. Viele Frauen klagen häufig nach Therapieabschluss über } \\
\text { Scheidentrockenheit, kennen Sie dieses Problem auch? }\end{array}$ \\
\hline $\begin{array}{l}\text { 6. Hat sich seit der Erkrankung etwas in Ihrer Partnerschaft, } \\
\text { insbesondere in der Sexualität verändert? }\end{array}$ \\
$\begin{array}{l}\text { 7. Wie geht es Ihnen mit der operierten Brust? } \\
\text { 8. Fällt es Ihnen schwer, sich unbekleidet vor Ihrem Partner } \\
\text { zu zeigen? }\end{array}$ \\
\hline
\end{tabular}

gen. Hinweise zu speziellen Gleitgels, fetthaltigen Hautpflegeprodukten und Informationsvermittlung helfen die Schamschwelle abzubauen und können einen offenen Umgang mit dem Tabuthema auch mit dem Partner erleichtern. Bei einer starken Selbstwertproblematik aufgrund des veränderten Körper- oder Selbstbilds sowie bei psychogenen sexuellen Funktionsstörungen sollte an einen niedergelassenen Psychotherapeuten oder Sexualtherapeuten verwiesen werden ( $\bullet$ Tab. 2).

\section{Kinderwunsch}

Besteht ein Kinderwunsch, sollte dies von vornherein in die Therapieplanung des Mammakarzinoms einbezogen werden. Es sollte eine erste Beratung bereits vor Beginn der Therapie erfolgen, um über Möglichkeiten der Ovarprotektion und Erhaltung der Fertilität aufzuklären. Laut aktuellen Leitlinien soll die prämenopausale Patientin mit vorhandenem Kinderwunsch, nach erfolgreichem Abschluss der Primärtherapie eines Mammakarzinoms, von professionellen Fachleuten über die Möglichkeiten der weiteren Familienplanung zeitnah aufgeklärt werden [14]. Kinderwunsch nach Krebs ist für die Betroffenen häufig ein schwieriges und kontrovers erlebtes Thema. Der Schock der Erkrankung und die Erschöpfung aufgrund der invasiven und z. T. aggressiv erlebten Therapien sind noch allgegenwärtig und trotzdem ist ein Wunsch nach einem (weiteren) Kind bei betroffenen Frauen sehr präsent. Viele Frauen sind nach $A b-$ schluss der Therapien verunsichert, ob eine (erneute) Empfängnis und Geburt eines Kindes ihr Rezidivrisiko erhöht oder dass ein Kind durch die aggressiven Therapien im Nachhinein geschädigt werden könnte [15]. Deswegen kann es wichtig sein, bei Patientinnen Mythen abzubauen und sie ggf. in ihrem Kinderwunsch zu bestärken, um den „optimalen Zeitpunkt“ nicht zu verpassen und um „unnötige Wartezeiten" zu verhindern. Ein Kinderwunsch sollte in das Therapie- und Nachsorgeprozedere aktiv integriert werden.

Adoption ist zwar für viele Paare anfangs nach einer schweren Erkrankung eine gute Option, obwohl der Wunsch nach einem biologischen Kind weiterhin sehr stark ausgeprägt ist [15]. Dabei gilt zu beachten, dass in Deutschland nach einer überstandenen Krebsdiagnose, auch nach der 5Jahres-Frist, eine Adoption eines Kindes aufgrund der Kindeswohlerwägungen meist nicht möglich ist. Zusätzlich muss berücksichtigt werden, dass das Adoptionsalter der Eltern laut Gesetz die 40 Jahre nicht überschreiten darf ( $\bullet$ Tab. 3 ).

\section{Umgang mit Kindern}

Durch Folgen der Erkrankung und Behandlung werden viele alte Rollen wie z.B. die Mutterrolle infrage gestellt. Viele Frauen machen sich nach Abschluss der Therapien starke Vorwürfe, dass sie ihre Kinder zu sehr mit ihrer Erkrankung belastet ha- 
- Tab. 3 Hilfreiche Fragen zum Thema Kinderwunsch.

\begin{tabular}{|l|l|}
\hline Ziele & mögliche Fragen \\
\hline $\begin{array}{l}\text { Klärung und Förderung } \\
\text { einer offenen Kommuni- } \\
\text { kation }\end{array}$ & $\begin{array}{l}\text { 1. Haben Sie sich schon Gedanken gemacht, ob Sie nach Ab- } \\
\text { schluss der Therapie (noch) ein Kind bekommen möchten? }\end{array}$ \\
$\begin{array}{l}\text { 2. Sie haben vor der Therapie erwähnt, dass Sie gerne noch } \\
\text { Kinder haben möchten. Wie sieht Ihre Familienplanung } \\
\text { aktuell aus? }\end{array}$ \\
\hline $\begin{array}{l}\text { Normalisierung und An- } \\
\text { sprechen der Mythen }\end{array}$ & $\begin{array}{l}\text { 3. Viele Frauen machen sich nach einer Krebserkrankung Ge- } \\
\text { danken darüber, ob der Körper noch in der Lage ist, ein Kind } \\
\text { zu empfangen. Kennen Sie solche Gedanken auch? }\end{array}$ \\
$\begin{array}{l}\text { 4. Einige Frauen befürchten, dass das Kind aufgrund der ver- } \\
\text { gangenen Therapien negative Konsequenzen erfährt. Geht } \\
\text { es Ihnen auch so? }\end{array}$ \\
$\begin{array}{l}\text { 5. Häufig haben Frauen Angst, aufgrund der schwanger- } \\
\text { schaftsbedingten Hormone erneut zu erkranken. Denken Sie } \\
\text { darüber aktuell nach? }\end{array}$ \\
\hline
\end{tabular}

- Tab. 4 Hilfreiche Fragen zum Thema Umgang mit Kindern.

\begin{tabular}{|l|l|}
\hline Ziele & mögliche Fragen \\
\hline $\begin{array}{l}\text { Klärung und Förderung } \\
\text { einer offenen Kommunika- } \\
\text { tion innerhalb der Familie }\end{array}$ & $\begin{array}{l}\text { 1. Welche „Informationspolitik“ wird bei Ihnen in der Familie } \\
\text { bez. der Erkrankung gemacht? }\end{array}$ \\
\hline $\begin{array}{l}\text { Legitimierung eigener } \\
\text { Gefühle und Bedürfnisse } \\
\text { sowie die der Kinder }\end{array}$ & $\begin{array}{l}\text { 3. Sprechen Sie in der Familie über die Gefühle? } \\
\text { 4. Kann Ihr Kind seine Gefühle bez. Ihrer Krankheit ausdrücken? } \\
\text { 5. Können eigene Bedürfnisse seitens Ihres Kindes ausgedrückt } \\
\text { werden? }\end{array}$ \\
\hline Ansprechen von Ressourcen & $\begin{array}{l}\text { 6. Was macht Ihr Kind, um mit Belastungen zurecht- } \\
\text { zukommen? }\end{array}$ \\
\hline $\begin{array}{l}\text { 7. Gibt es noch weitere Bezugspersonen außerhalb der Familie, } \\
\text { die sich um die Kinder kümmern können und die als An- } \\
\text { sprechpartner zur Verfügung stehen? }\end{array}$
\end{tabular}

ben und dass sie aufgrund dessen eine schlechte Mutter gewesen seien. Sie haben das Gefühl, dass sie sich während der Therapiezeit zu wenig um ihre Kinder gekümmert haben und dass die Kinder nun nicht mehr unbeschwert aufwachsen können. Unsicherheiten, Schuld- und Schamgefühle seitens der Mütter bzw. Eltern führen häufig dazu, dass wenig über die Erkrankung innerhalb der Familie, auch nach Abschluss der Akutbehandlung, gesprochen wird [16]. Darüber hinaus leiden Kinder nach einer überstandenen Behandlung der Mutter immer wieder unter Progredienz- oder Trennungsängsten. Sie haben durch die therapiebedingten Veränderungen wie z.B. eine krank erlebte Mutter, ein belasteter Vater oder Betreuung durch andere Personen, Verunsicherung innerhalb des Familiensystems erfahren. Jedoch zeigen Studien, dass entscheidende Schutzfaktoren für die psychische Belas- tung der Kinder krebskranker Eltern, zum einen die offene Kommunikation innerhalb der Familie und zum anderen der emotionale Ausdruck der eigenen Empfindungen bez. der Erkrankung, hilfreich sind $[17,18]$. Allerdings ist bei letzterem darauf zu achten, dass Kinder nicht zu sehr mit den Emotionen Erwachsener „überflutet“ werden, damit diese nicht die Rolle der Tröster und Kümmerer einnehmen. Trotzdem können und sollen Kinder durch das aktive Einbeziehen in die aktuelle Situation in ihren Bewältigungsmechanismen bestärkt werden und so lernen, ihren eigenen Weg mit der Erkrankung der Mutter zu gehen. Nichtsdestotrotz fühlen sich viele Eltern unsicher, wie sie offen und kindgerecht über die aktuelle Erkrankungssituation sprechen sollen und wünschen sich hierbei häufig eine professionelle Beratung bzw. Unterstützung [19] (॰ Tab. 4).
FAZIT

Nach Abschluss der Akutbehandlung weisen viele Patientinnen mit Mammakarzinom psychosoziale Belastungen und Bedürfnisse in verschiedenen psychoonkologischen und gynäkologischen Themenbereichen auf. Patientinnen wünschen sich in der Nachbehandlung, dass diese Belastungen und Themen wie Sexualität und Kinderwunsch aktiv von behandelten Ärzten auch in der Nachsorge angesprochen werden. Durch die offene Kommunikation sowie Normalisierung und Enttabuisierung der Belastungsbereiche können Patientinnen in ihren psychosozialen Bedürfnissen erreicht werden und auf mögliche Unterstützungsangebote (Ratgeber, Webseiten, Therapeuten etc.; siehe Infobox) aufmerksam gemacht werden. Dadurch ist es ihnen möglich, eine adaptive Krankheitsbewältigung durch eigene Ressourcen und Kompetenzen nach überstandener Krebsdiagnose und -behandlung zu entwickeln.

\section{Interessenkonflikt}

Die Autoren geben an, dass kein Interessenkonflikt besteht.

Über die Autoren

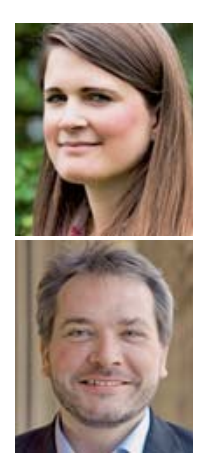

Johanna Ringwald

Abteilung für Psychosomatische Medizin und Psychotherapie, Medizinische Klinik, Universitätsklinikum Tübingen

\section{Martin Teufel}

Abteilung für Psychosomatische Medizin und Psychotherapie, Medizinische Klinik, Universitätsklinikum Tübingen und Klinik für Psychosomatische Medizin und Psychotherapie am LVR-Klinikum Essen, Universität Duisburg-Essen

\section{Korrespondenzadresse}

Johanna Ringwald,

Dipl.-Psych.; Psychoonkologin

Universitätsklinikum Tübingen, Medizinische Klinik, Abteilung für Psychosomatische Medizin und Psychotherapie

Osianderstraße 5

72076 Tübingen

Johanna.Ringwald@med.uni-tuebingen.de 


\section{ZUSATZINFORMATION}

\section{Hilfreiches Material und Webseiten}

Webseiten, z. B.

- www.krebsinformationsdienst.de

- www.krebshilfe.de

- www.infonetz-krebs.de

- www.inkanet.de

- www.du-bist-kostbar.de

- www.fertiprotekt.com

- www.mum-hat-brustkrebs.de

- www.psychotherapeutensuche.de

- www.dgsmtw.de

Listen von niedergelassenen und wohnortnahen Psychotherapeuten können auch über die Ärztekammern der jeweiligen Bundesländer aufgerufen werden. Eine übersichtliche Liste von wohnortnahen Krebsberatungsstellen und Psychoonkologen kann z. B. der Krebsinformationsdienst zur Verfügung stellen.
Ratgeber, z. B.

- Weibliche Sexualität und Krebs ein Ratgeber für Patientinnen und ihre Partner vom Krebsinformationsdienst

- Krebspatientin und Sexualität ein Ratgeber für Betroffene und Angehörige von der Südtiroler Krebshilfe in Zusammenarbeit mit dem Krebsinformationsdienst

- Kinderwunsch nach Krebs die blauen Ratgeber der deutschen Krebshilfe und deutschen Krebsgesellschaft

- Mit Kindern über Krebs sprechen ein Ratgeber für Eltern, die an Krebs erkrankt sind

- Was jetzt für Dich wichtig ist ein Ratgeber von und für Jugendliche, deren Eltern an Krebs erkrankt sind
Kinderbücher, z. B.

- Der Chemo-Kasper ein Bilderbuch für kleine Kinder über Krebs und Chemotherapie

- Radio-Robby ein Bilderbuch für kleine Kinder über Krebs und Strahlentherapie

- Manchmal ist Mama müde ein Kinderbuch zum Thema Brustkrebs

\section{App für das Smartphone \\ - Der Zauberbaum - unterstützt die Eltern-Kind- Kommunikation}

\section{Literatur}

[1] Robert Koch-Institut; Gesellschaft der epidemiologischen Krebsregister in Deutschland e.V., Hrsg. Krebs in Deutschland 2011/ 2012. 10. Ausgabe. Berlin; 2015

[2] Lee ES, Lee MK, Kim SH et al. Health-related quality of life in survivors with breast cancer 1 year after diagnosis compared with the general population: a prospective cohort study. Ann Surg 2011; 253: 101-108

[3] Partridge $\mathrm{AH}$. Cancer survivorship and the young breast cancer patient: addressing the important issues. Oncologist 2013; 18: e19e20

[4] Mehnert A, Brähler E, Faller $\mathrm{H}$ et al. Fourweek prevalence of mental disorders in patients with cancer across major tumor entities. J Clin Oncol 2014; 32: 3540-3546

[5] Mitchell AJ, Ferguson DW, Gill J et al. Depression and anxiety in long-term cancer survivors compared with spouses and healthy controls: a systematic review and meta-analysis. Lancet Oncol 2013; 14: 721-732

[6] Ahmad S, Fergus K, McCarthy M. Psychosocial issues experienced by young women with breast cancer: the minority group with the majority of need. Curr Opin Support Palliat Care 2015; 9: 271-278

[7] Havighurst RJ. Human Development and Education. London: Longmans; 1953
[8] Schmid-Büchi S, Dassen T, Halfens R]. Die Erfahrung, an Brustkrebs zu erkranken, und wie die betroffenen Frauen ihr Leben wieder unter Kontrolle bringen: Experiencing the disease of breast cancer and getting life under control again. Pflege 2005; 18: 345-352

[9] Campbell-Enns HJ, Woodgate RL. The psychosocial experiences of women with breast cancer across the lifespan: a systematic review. Psychooncology 2016; DOI: 10.1002 | pon. 4281

[10] Taylor S, Harley C, Absolom K et al. Breast cancer, sexuality, and intimacy: addressing the unmet need. Breast J 2016; 22: 478-479

[11] Kedde $\mathrm{H}$, van de Wiel HB, Weijmar Schultz WC et al. Sexual dysfunction in young women with breast cancer. Support Care Cancer 2013; 21: 271-280

[12] Dadak C, Sohlmann S. Sexualität nach einer Krebserkrankung. Der Gynäkologe 2014; 47: 99-103

[13] Paterson CL, Lengacher CA, Donovan KA et al. Body image in younger breast cancer survivors: a systematic review. Cancer Nurs 2016; 39: E39-E58

[14] Leitlinienprogramm Onkologie der AWMF, Deutschen Krebsgesellschaft e.V. und Deutschen Krebshilfe e.V., Hrsg. Interdisziplinäre S3-Leitlinie für die Diagnostik, Therapie und Nachsorge des Mammakarzinoms: Langversion 3.0, Aktualisierung 2012, AWMF-Register-Nummer: 032-045OL. Germering: Zuckschwerdt; 2012
[15] Gonçalves V, Sehovic I, Quinn G. Childbearing attitudes and decisions of young breast cancer survivors: a systematic review. Hum Reprod Update 2013; 20: 279-292

[16] Semple C], McCance T. Parents' experience of cancer who have young children: a literature review. Cancer Nurs 2010; 33: 110-118

[17] Asbury N, Lalayiannis L, Walshe A. How do I tell the children? Women's experiences of sharing information about breast cancer diagnosis and treatment. Eur J Oncol Nurs 2014; 18: 564-570

[18] Howell KH, Barrett-Becker EP, Burnside AN et al. Children facing parental cancer versus parental death: the buffering effects of positive parenting and emotional expression. I Child Fam Stud 2016; 25: 152-164

[19] Götze H, Zenger M, Brähler E et al. Brustkrebspatientinnen mit minderjährigen Kindern - Psychosoziale Belastung und Unterstützungswünsche. Z Klin Psychol Psychiatr Psychother 2015; 62: 55-63

[20] Rowland JH, Massie M]. Breast Cancer. In: Holland JC, Breitbart W, Jacobsen PB, eds. Psycho-oncology. 3rd ed. New York: Oxford University Press; 2010: 177-186

\section{Bibliografie}

DOI http://dx.doi.org/10.1055/s-0042-124585 Geburtsh Frauenheilk 2017; 77: 465-470 @ Georg Thieme Verlag KG Stuttgart · New York I ISSN 0016-5751 


\section{Der Beitrag wurde gemäß folgendem Erratum korrigiert:}

Im Beitrag „Psychosoziale Herausforderungen und Bedürfnisse junger Frauen nach therapiertem Mammakarzinom“ (Geburtsh Frauenheilk 2017; 77: 465-469) lautet der korrekte Titel: „Psychosoziale Herausforderungen und Bedürfnisse junger Frauen nach onkologischer Behandlung eines Mammakarzinoms“. Im ersten Absatz des Abschnitts „Hintergrund“ lautet der zweite Satz korrekt: „Es wird geschätzt, dass 2016 etwa 65500 Frauen neu erkrankt sind, was konkret bedeutet, dass jede 8. Frau im Laufe ihres Lebens an Brustkrebs erkrankt.“ Die Affiliation beim Autor Martin Teufel lautet vollständig: Abteilung für Psychosomatische Medizin und Psychotherapie, Medizinische Klinik, Universitätsklinikum Tübingen und Klinik für Psychosomatische Medizin und Psychotherapie am LVR-Klinikum Essen, Universität Duisburg-Essen. 